\title{
Ultrasonido y anestesia: ¿El estetoscopio de los "Millennials"?
}

La ecocardiografía comenzó a desarrollarse a partir de la década de 1950, producto del esfuerzo conjunto de diversos investigadores suecos, entre los que destacan Edler y Hertz ${ }^{1}$. La tecnología inicial fue la ecocardiografía transtorácica (ETT), que estuvo confinada por un tiempo al área del diagnóstico en cardiología pero con la aparición de la ecocardiografía transesofágica (ETE) en 1980 fue exitosamente incorporada al ámbito de la cirugía cardíaca a fines de la misma década, pues permitía la visualización del corazón sin interferir con el campo quirúrgico². En la actualidad su uso en cirugía cardíaca sería prácticamente mandatorio como es el caso de la cirugía valvular ${ }^{3,4}$. En cirugía no cardiaca el uso intraoperatorio de la ETE no está tan firmemente arraigado pero las recomendaciones de la Asociación Americana de Anestesiólogos $(A S A)^{5}$, sugieren su utilización en toda situación de hipotensión e hipoxemia inexplicada y refractaria o cuando se anticipa que alguna de estas situaciones catastróficas podrían ocurrir como por ejemplo en un trasplante pulmonar.

La necesidad de extender la monitorización hemodinámica al pre y post-operatorio ${ }^{6}$, períodos en los cuales no se podía recurrir a la ETE, hizo volver la mirada nuevamente hacia la ETT. Dado su carácter no invasivo y portátil, ésta comenzó a ser usada por anestesiólogos, intensivistas y médicos de urgencia ${ }^{7}$ "al lado de la cama de paciente" 8 . La versatilidad de sus beneficios incluyen: ágil evaluación preoperatoria de pacientes considerados de alto riesgo y sometidos a cirugías de urgencia absoluta o relativa ${ }^{4,9}$, en las que no hay tiempo para pedir una evaluación formal por un cardiólogo, determinar si existe neumotórax en un poli-traumatizado ${ }^{8}$ y quizás lo más importante: en el diagnóstico diferencial y manejo del colapso hemodinámico ("shock") 10,11. En todas estas situaciones la ETT ofrece la posibilidad de responder certeramente a preguntas específicas, enfocadas ("focused") en el problema, en donde sea que nos encontremos: Pabellón, Unidad de Recuperación, Servicio de Urgencia, $\mathrm{UCl}$, helicóptero de rescate, sin necesariamente trasladar al paciente a una unidad de radiología (de ahí el nombre "POCUS" "Point-of-Care UltraSound")7,8,12,13 y así tomar decisiones ${ }^{14}$ que determinaran cambios en la conducta terapéutica en el $16-58 \%$ de los pacientes ${ }^{15}$.

La deuda pendiente sería demostrar que estas nuevas aplicaciones de la ultrasonografía tienen un impacto medible en cuanto a mejorar el pronósti$\mathrm{Co}^{16}$. Este efecto probablemente nunca será calculado puesto que nadie privaría a un paciente en colapso circulatorio de los beneficios que ofrece un método no invasivo con tanta riqueza de información diagnóstica.

Pero la utilidad del ultrasonido en el período perioperatorio no se ha limitado a la ecocardiografía; durante los últimos 20 años ha influenciado la práctica de la anestesia regional ${ }^{17}$ y desde hace 10 años es considerado un estándar para la instalación de accesos vasculares ${ }^{18}$.

En este número la Dra. Carolina Cabrera en conjunto con el grupo de expertos de la Asociación de Ecocardiografía e Imagen Cardiovascular de la Sociedad Interamericana de Cardiología (ECOSIAC), presentan las primeras recomendaciones latinoamericanas para el uso de la ecocardiografía perioperatoria ${ }^{4}$, las cuales, como la misma Dra. Cabrera lo expresa, no constituyen una guía institucional sino solo una manera de ordenar el conocimiento que existe hasta la fecha y aplicarlo a nuestra realidad.

El Dr. Luis Azócar también, nos describe estas nuevas aplicaciones del ultrasonido 8 .

Se agradece a ambos el esfuerzo de exponer y organizar los beneficios de esta metodología que llegó primero a la cardio-anestesia pero que actualmente permeó todas las áreas de nuestra especialidad, desde el pre hasta el postoperatorio.

Dagoberto Ojeda D. Editor

Servicio Anestesiología Clínica Dávila Av. Recoleta 464, $3^{\text {er }}$ Piso, Edificio D E-mail: eojedadinamarca@gmail.com 


\section{Referencias}

1. Edler I, Lindström K. The history of echocardiography. Ultrasound Med Biol. 2004 Dec;30(12):1565-644.

2. Kneeshaw JD. Transoesophageal echocardiography (TOE) in the operating room. Br J Anaesth. 2006 Jul;97(1):77-84.

3. Klein AA, Snell A, Nashef SA, Hall RM, Kneeshaw JD, Arrowsmith JE. The impact of intraoperative transoesophageal echocardiography on cardiac surgical practice. Anaesthesia. 2009 Sep;64(9):947-52.

4. ECOSIAC. (Asociación de Ecocardiografía e Imágen Cardiovascular de la Sociedadinteramericana de cardiología). Grupo de Trabajo en Ecocardiografia Perioperatoria. María Carolina Cabrera S, Iván Iglesias, Mariano Falconi, Rodrigo Hernández $\checkmark$,Salvador Spina, Marcelo Luiz Campos V. Primeras recomendaciones clínicas latinoamericanas para ecocardiografía perioperatoria. Rev Chil Anest. 2017;46:142-52.

5. American Society of Anesthesiologists and Society of Cardiovascular Anesthesiologists Task Force on Transesophageal Echocardiography. Practice guidelines for perioperative transesophageal echocardiography. An updated report by the American Society of Anesthesiologists and the Society of Cardiovascular Anesthesiologists Task Force on Transesophageal Echocardiography. Anesthesiology. 2010 May;112(5):1084-96.

6. Jørgensen MR, Juhl-Olsen P, Frederiksen CA, Sloth E. Transthoracic echocardiography in the perioperative setting. Curr Opin Anaesthesiol. 2016 Feb;29(1):4654.

7. Neskovic AN, Edvardsen T, Galderisi M, Garbi M, Gullace G, Jurcut R et al.; European Association of Cardiovascular Imaging Document Reviewers. Focus cardiac ultrasound: the European Association of Cardiovascular Imaging viewpoint. Eur Heart J Cardiovasc Imaging. 2014 Sep;15(9):956-60.

8. Azócar L. Uso de ecografía en anestesia: Point of Care Ultrasound (POCUS). Rev Chil Anestesia. 2017;46:153-62.

9. Canty DJ, Royse CF. Audit of anaesthetist-performed echocardiography on perioperative management decisions for noncardiac surgery. Br J Anaesth. 2009 Sep;103(3):352-8.

10. Vincent JL, De Backer D. Circulatory shock. N Engl J Med. 2013 Oct;369(18):1726-34.

11. Mok KL. Make it SIMPLE: enhanced shock management by focused cardiacultrasound. J Intensive Care. 2016 Aug 15;4:51.

12. Moore CL, Copel JA. Point-ofcare ultrasonography. N Engl J
Med. 2011 Feb;364(8):749-57.

13. Peterson D, Arntfield RT. Critical care ultrasonography. Emerg Med Clin North Am. 2014 Nov;32(4):907-26.

14. International Liaison Committee on Focused Cardiac UltraSound (ILC-FoCUS) for the International Conference on Focused Cardiac UltraSound (IC-FoCUS). International Evidence-Based Recommendations for Focused Cardiac Ultrasound. J Am Soc Echocardiogr. 2014;27:683.e1-e33.

15. Kratz $T$, Steinfeldt $T$, Exner $M$, Dell Orto MC, Timmesfeld N, Kratz $C$ et al. Impact of Focused Intraoperative Transthoracic Echocardiography by Anesthesiologists on Management in Hemodynamically Unstable HighRisk Noncardiac Surgery Patients. J Cardiothorac Vasc Anesth. 2017 Apr;31(2):602-9.

16. Wetterslev M, Haase N, Johansen RR, Perner A. Predicting fluid responsiveness with transthoracic echocardiography is not yet evidence based. Acta Anaesthesiol Scand. 2013 Jul;57(6):692-7.

17. Marhofer P, Harrop-Griffiths W, Kettner SC, Kirchmair L. Fifteen years of ultrasound guidance in regional anaesthesia: part 1. Br J Anaesth. 2010 May; 104(5):53846.

18. Reusz G, Csomos A. The role of ultrasound guidance for vascular access. Curr Opin Anaesthesiol. 2015 Dec;28(6):710-6. 